Journal of Applied Fluid Mechanics, Vol. 15, No. 1, pp. 209-220, 2022.

Available online at www.jafmonline.net, ISSN 1735-3572, EISSN 1735-3645.

https://doi.org/10.47176/jafm.15.01.33041

\title{
On the Scale Size of the Aerodynamic Characteristics of a High-Speed Train
}

\author{
C. Chang, T. Li ${ }^{\dagger}$, D. Qin and J. Zhang \\ State Key Laboratory of Traction Power, Southwest Jiaotong University, Chengdu610031, China \\ $\dagger$ Corresponding Author Email: litian2008@home.swjtu.edu.cn
}

(Received May 25, 2021; accepted September 4, 2021)

\begin{abstract}
In the wind tunnel test of trains, the scale size changes the Reynolds numbers of trains, which may affect the aerodynamic characteristics of the train. Based on computational fluid dynamics (CFD), numerical models of train aerodynamics with five different scale sizes are established. The five different scale sizes are $\lambda=1 / 1,1 / 2$, $1 / 8,1 / 16$ and $1 / 25$, respectively, and the aerodynamic characteristics of trains running in the open-air operating condition and crosswind operating condition with different scale sizes are numerically simulated. The results show that the pressure drag coefficients and pressure lift coefficients of the train tend to decrease with the decrease of the scale size. In the open-air operating condition, compared with the full-size train, the pressure drag coefficient of the $1 / 25^{\text {th }}$ scaled train is less by $14.4 \%$, and the pressure lift coefficients of the head car, middle car and tail car change $16.1 \%, 46.6 \%$ and $12.3 \%$, respectively. The scale size affects the velocity gradient near the train surface and the position of flow separation changes. The decrease of the scale size leads to the decrease of Reynolds numbers and the increase of viscous drag coefficient. When the scale size is $1 / 25$, the viscous drag coefficient of the train is 0.186 , which is $48.6 \%$ larger than the one of the full-size train. Compared with the open-air operating condition, the trend of the pressure drag coefficients and viscous drag coefficients is consistent except for the head car in crosswind operating condition when the scale size decreases. In the range of scale size $\lambda$ between $1 / 1$ and $1 / 25$, the aerodynamic drag coefficient of the head car, middle car and tail car increase with the decrease of scale size, and the difference in the aerodynamic drag coefficient of the train is $12.9 \%$. In addition, the train's aerodynamic lift coefficient shows an increasing trend with the decrease of scale size.
\end{abstract}

Keywords: CFD; Scale size; High-speed trains; Aerodynamic force coefficient.

\section{NOMENCLATURE}

$\begin{array}{ll}A & \text { characteristics area of the train } \\ C_{d} & \text { aerodynamic drag force coefficient } \\ C_{d, p} & \text { pressure drag force coefficient } \\ C_{d, v} & \text { viscous drag coefficient } \\ C_{l} & \text { aerodynamic lift coefficient } \\ C_{l, p} & \text { pressure lift coefficient } \\ C_{l, v} & \text { viscous drag coefficient } \\ H_{t} & \text { characteristic height of the train } \\ H_{m} & \text { base size of the mesh } \\ F_{x} & \text { aerodynamic drag }\end{array}$

\section{INTRODUCTION}

Aerodynamic characteristics of high-speed trains have become a hot research topic in recent years, especially with the increasing running speed of trains (Yu et al. 2021). Due to the shape of the head car, various operating conditions and the high turbulence,

$\begin{array}{ll}F_{z} & \text { aerodynamic lift } \\ p & \text { static pressure } \\ R e & \text { Reynolds number } \\ u_{\infty} & \text { velocity at the inlet boundary } \\ v & \text { running speed of trains } \\ \lambda & \text { scale size } \\ \mu & \text { air dynamic viscosity } \\ \rho & \text { air density } \\ \sigma & \text { thickness of boundary layer }\end{array}$

the flow around the high-speed trains is complex. ( $\mathrm{Lu}$ et al. 2012; Zhang et al. 2017; Li et al. 2017). At present, the main research methods for the train aerodynamics include theoretical analysis, wind tunnel tests and numerical simulation (Li et al. 2021). The environmental conditions of full-scale train tests are not easy to control, and weather can greatly affect 
the flow field. Numerical simulations have been widely used to evaluate the aerodynamic characteristics of trains (Li et al. 2020). The results obtained using numerical simulations should be verified and corrected by wind tunnel tests (Emil et al. 2020).

The wind tunnel test is based on the principle of relativity of motion, which makes the air flow over a stationary train at a specific speed to obtain the aerodynamic forces of the trains. In wind tunnel tests, several similarity criterions are required to ensure the correctness of experimental results. During the operation of the high-speed trains, the viscous forces play a dominant role and the Reynolds numbers of the scaled model should be consistent. However, due to the limitations of actual conditions, such as the size of the wind tunnel, cost and so on, it is almost impossible to achieve a full-scale train in the wind tunnel (Collin et al. 2016). At present, it is generally believed that the factors affecting the accuracy of wind tunnel tests are ground conditions, blocking ratio, equipment settings, length of the test section, and the effect of Reynolds numbers (Kwon et al. 2001; Bell et al. 2017). When conducting wind tunnel tests for the high-speed trains, all parameters are generally kept constant except for the scale size, therefore the Reynolds number effect is also called the scale effect.

The error in the aerodynamic coefficients induced by the scale size is much larger than that caused by various ground conditions (Baker and Brockie 1991). The effect of train aerodynamics and pressure in the range of Reynolds number from $3.02 \times 10^{5}$ to $2.27 \times 10^{6}$ is observed by experiments, and the positive and negative pressure zones on the train surface have different variation patterns (Niu et al. 2016).

The changes in aerodynamic characteristics are generally due to changes in the surrounding flow field. At present, the knowledge on the influence of Reynolds numbers on the flow field is mainly concentrated on simple structures, such as cylinder, flat plate and rectangular structures. The turbulent boundary layer of a flat plate with a Reynolds number ranging from 2600 to 72,500 is observed by Vallikivi et al. (2015). When the Reynolds number was larger than 20,000, the flow showed similar characteristics. De Graaff et al. (2000) observed and analyzed the turbulent flow of flat plates with Reynolds numbers ranging from 1430 to 31000 , and the experimental results showed that the logarithmic method can provide a fairly accurate inference for the velocity distribution inside the turbulent boundary layer. It was concluded that for the flow around a circular cylinder, the aerodynamic drag coefficient is a function of the Reynolds number (Catalano et al. 2003). For the train, the Reynolds number shows a self-similarity within a certain range (Bell et al. 2014). Under such conditions, the aerodynamic characteristics of the train are less affected by the Reynolds number (Zhou et al. 2014). According to EN14067-6, the Reynolds number in the wind tunnel experiment shall be greater than $2,5 \times 10^{5}$ to ensure flow similarity between model and full-scale flows. However, there has been no definite conclusion on the extent and impact of self-similarity, and whether it can be applied in actual trains (Paz et al. 2015). For a high-speed train, the Reynolds number of a fullscale or $1 / 25^{\text {th }}$ scaled train is in the range of $10^{5}$ and $10^{7}$, which may greatly affect the flow around a highspeed train. Up to now, few researches have focused on the Reynolds number with such a large range of the aerodynamic characteristics of a high-speed train.

This study focuses on not only the influence of the scale size on the aerodynamic drag coefficient of the train but also the aerodynamic lift coefficient. In addition, the trend of train pressure force and viscous force affected by the scale size in open-air and crosswind operating conditions are discussed. Numerical simulations of trains with five different scale sizes including $\lambda=1 / 1,1 / 2,1 / 8,1 / 16$ and $1 / 25$ are conducted to compare and analyze the air flow field around the high-speed trains. The results provide a reference for the correction of wind tunnel test results.

The present paper is structured as follows. Section 2 describes the numerical information including the turbulent model, a simplified train and the numerical model. Section 3 reports the comparisons of aerodynamic characteristics between different scale sizes. And conclusions are drawn in Section 4.

\section{NUMERICAL INFORMATION}

\subsection{Turbulent model}

As the running speed $v$ of a train is $300 \mathrm{~km} / \mathrm{h}$ in the open-air condition, the flow can be considered as an incompressible one, therefore, the air density $\rho$ is constant, $\rho=1.225 \mathrm{~kg} / \mathrm{m}^{3}$. For an incompressible flow, the SST (shear stress transport) $k$ - $\omega$ turbulence model is a valid tool. It can accurately solve the viscous sublayers near the wall, and the accuracy of the flow near the train surface is high. It is widely used in the numerical simulation of train aerodynamics and provides the numerical results of aerodynamic forces in good agreement with the wind tunnel test results (Li et al. 2019a).

\subsection{Geometric model}

Figure 1 shows the train model. It is a Chinese highspeed train with a head car, a middle car and a tail car. The height of the trains chosen as the characteristic height $H_{\mathrm{t}}$ according to the hydraulic diameter. $A$ is the characteristics area of the train. The distance between the bottom of the train and the roadbed is $425 \mathrm{~mm}$.
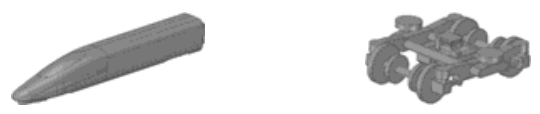

(a)Head car

(b)Bogie

Fig. 1. Geometric model. 
The center point of the train is the origin of the coordinate system.

\subsection{Numerical Details}

Figure 2(a) shows the calculation domain of the numerical simulation for the open-air operating condition. The inlet boundary is $100 \mathrm{~m}$ far from the nose of the head car and the incoming velocity is the running speed of the train $v=83.3 \mathrm{~m} / \mathrm{s}$. The outlet boundary is $200 \mathrm{~m}$ far from the nose of the tail car and a pressure outlet condition that is prescribed with a pressure of zero. The top and side boundaries are set as symmetric conditions. The train surface is set as a non-slip fixed wall. To truly simulate the wind tunnel test, the ground and embankment are also set as fixed walls. Figure 2(b) shows the calculation domain for crosswinds. The boundary condition of inlet 1 and inlet 2 are velocity-inlet. The outlet 1 and outlet2 boundaries are pressure outlet boundaries that are prescribed with a pressure of zero. The speed of wind is $20 \mathrm{~m} / \mathrm{s}$. SIMPLE algorithm is used to calculate the pressure and velocity, and all variables are in secondorder format.

In the wind tunnel test, the scale size $\lambda$ of trains are generally $1 / 8$ or $1 / 25$. A $1 / 25$ th-scale train experiment of an Inter-City Express (ICE3) was operated to obtain aerodynamics (Bell et al. 2017); a series of wind tunnel tests were conducted on Korean highspeed train models with scale size of $1 / 25$ using various ground condition simulation techniques (Kwon et al. 2001); scholars have also conducted a lot of research on the aerodynamic characteristics of trains with scale size $\lambda=1 / 8$ (Xia et al. 2017).

To better investigate the effect of the scale size on the aerodynamic characteristics of trains, in addition to the scale size $\lambda=1 / 1,1 / 8$ and $1 / 25$, numerical simulations are also performed for scale size $\lambda=1 / 2$ and $1 / 16$ trains.

Table 1 shows the Reynolds numbers for five different scaled trains. To keep the consistency between different scale sizes, the velocity and dynamic viscosity of air are consistent.

\subsection{Mesh sensitivity and validation}

The mesh around the train and boundary layers are shown in Fig. 3. To describe the mesh size, the maximum size of the mesh is chosen as the benchmark of the mesh size $H_{\mathrm{m}}$. The mesh size of the train surface is $\left(1 / 2^{7} \sim 1 / 2^{4}\right) H_{\mathrm{m}}$, the mesh size of the windshield surface is $\left(1 / 2^{7} \sim 1 / 2^{6}\right) H_{\mathrm{m}}$, and the mesh size of the bogie surface is $\left(1 / 2^{7} \sim 1 / 2^{6}\right) H_{\mathrm{m}}$, the growth rate of the surface mesh is 1.2. Meanwhile, four mesh encryption areas are set, and their mesh size are $H_{\mathrm{m}} / 2^{4}, H_{\mathrm{m}} / 2^{3}, H_{\mathrm{m}} / 2^{2}$, and $H_{\mathrm{m}} / 2^{1}$, respectively. The number of boundary layers is twelve. To ensure a good mesh transition of the boundary layer mesh and the $y+$ of the train is around one, the growth ratio between neighboring layers is 1.2 and the height of the first boundary layer is $0.015 \mathrm{~mm}$.

The aerodynamic force coefficients are chosen for comparison. The aerodynamic drag coefficient $C_{d}$ and the aerodynamic lift coefficient $C_{l}$ are defined as

$$
\begin{aligned}
C_{d} & =\frac{F_{x}}{0.5 \rho u_{\infty}^{2} A} \\
C_{l} & =\frac{F_{z}}{0.5 \rho u_{\infty}^{2} A}
\end{aligned}
$$

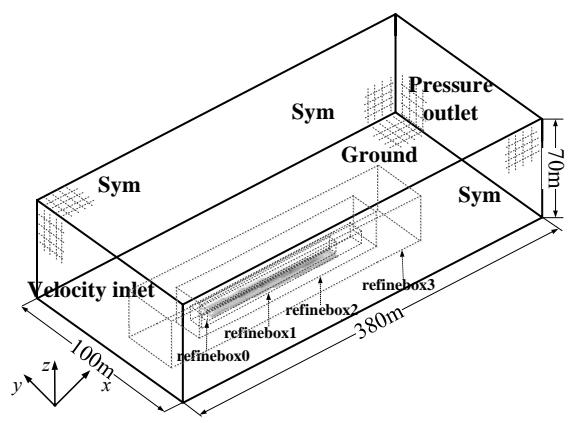

(a) Open-air operating condition

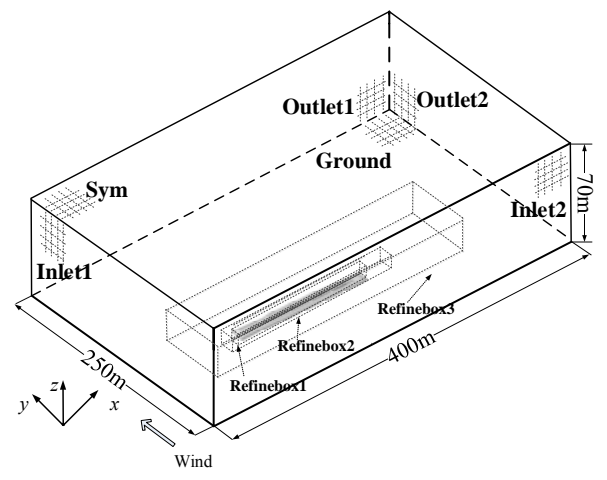

(b) Crosswind operating condition

Fig. 2. Computational domain and boundary conditions.

Table 1 Reynolds number of high-speed trains at different scale sizes

\begin{tabular}{|c|c|c|c|c|c|}
\hline$\lambda$ & $H_{\mathrm{t}}(\mathrm{m})$ & $A\left(\mathrm{~m}^{2}\right)$ & $u_{\infty}(\mathrm{m} / \mathrm{s})$ & $\mu\left(\mathrm{Ns} / \mathrm{m}^{2}\right)$ & $\operatorname{Re}$ \\
\hline $1 / 1$ & 3.7000 & 10.8000 & 83.3 & $1.46 \times 10^{-5}$ & $2.11 \times 10^{7}$ \\
\hline $1 / 2$ & 1.8500 & 2.7000 & 83.3 & $1.46 \times 10^{-5}$ & $1.06 \times 10^{7}$ \\
\hline $1 / 8$ & 0.4625 & 0.1687 & 83.3 & $1.46 \times 10^{-5}$ & $2.64 \times 10^{6}$ \\
\hline $1 / 16$ & 0.2313 & 0.0422 & 83.3 & $1.46 \times 10^{-5}$ & $1.32 \times 10^{6}$ \\
\hline $1 / 25$ & 0.1480 & 0.0173 & 83.3 & $1.46 \times 10^{-5}$ & $8.44 \times 10^{5}$ \\
\hline
\end{tabular}


Table 2 Results obtained by using different meshes

\begin{tabular}{|c|c|c|c|c|c|c|}
\hline Mesh & $H_{\mathrm{m}}(\mathrm{m})$ & Numbers of cells(million) & $C_{d \text {-head }}$ & $C_{d \text {-mid }}$ & $C_{d \text {-tail }}$ & $C_{d \text {-train }}$ \\
\hline Mesh1 & 2.2 & 22.96 & 0.126 & 0.080 & 0.104 & 0.310 \\
\hline Mesh2 & 1.9 & 34.19 & 0.121 & 0.085 & 0.101 & 0.307 \\
\hline Mesh3 & 1.6 & 41.09 & 0.122 & 0.084 & 0.100 & 0.306 \\
\hline
\end{tabular}

Where $F_{x}, F_{z}$ are the aerodynamic drag and aerodynamic lift respectively during the operation of the train, $\rho$ is the air density, $u_{\infty}$ is the velocity at the inlet boundary, and $A$ is the characteristics area of the train.

Three meshes, named Mesh1, Mesh2 and Mesh3, were generated according to different sizes of $H_{\mathrm{m}}$. The numerical results of the $1 / 8^{\text {th }}$ scaled model obtained by using the above meshes are compared with the wind tunnel test results. Table 2 shows the aerodynamic force coefficients obtained by using numerical simulation for different meshes. Compared with Mesh2 and Mesh3, the aerodynamic drag coefficient of Mesh1 has a large difference, The differences of head car, middle car and tail car between Mesh2 and Mesh3 are 0.83\%, 1.17\% and $0.99 \%$, respectively. The differences between Mesh2 and Mesh3 are less than $2 \%$, and Mesh2 has fewer cells, which is more efficient while satisfying the calculation accuracy. Therefore, Mesh2 is selected for calculation.

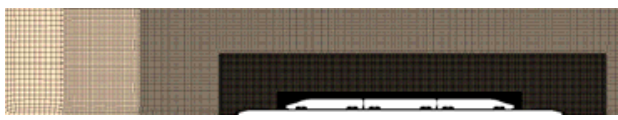

(a) Mesh around the train

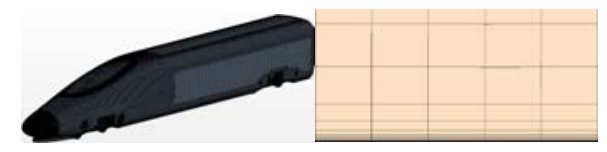

(b) Surface mesh of head car (c) Boundary layer mesh of the train

Fig. 3. Calculation mesh.

To verify the accuracy of the numerical calculation method, the numerical calculation results of Mesh2 are compared with the wind tunnel test result for verification (Han and Song 2017). The train models in the wind tunnel test and numerical simulation are both $1 / 8$ scaled. Figure 4 shows the comparison between the numerical calculation for Mesh2 and the wind tunnel test. The maximum difference of each drag coefficient is $7.25 \%$, the pressure coefficient of the stagnation point of the head car is 0.986 . The numerical calculation results are in good agreement with the wind tunnel test results, which proves the accuracy of the numerical calculation results.

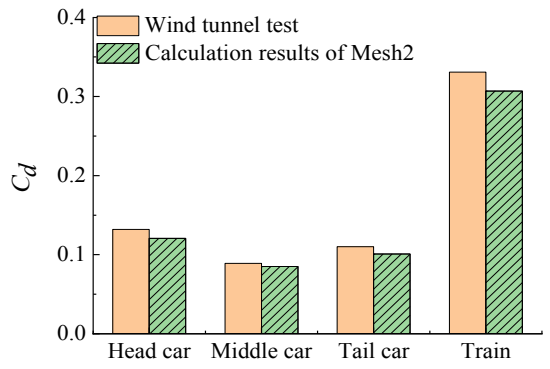

Fig. 4. Comparison of numerical calculation results with wind tunnel test results.

\section{Calculation results}

Numerical calculations are conducted for trains with scale size of $\lambda=1 / 1,1 / 2,1 / 8,1 / 16$ and $1 / 25$ for openair operation. The calculation results will be analyzed in terms of three aspects: surface pressure, the flow field around the train and aerodynamic force.

\subsection{Train surface pressure and flow field}

Figure 5 shows the surface pressure of the head car at different scale sizes. When the scale size $\lambda=1 / 1,1 / 2$, $1 / 8,1 / 16$ and $1 / 25$, the pressure coefficient of stagnation-point is $0.992,0.990,0.986,0.982,0.979$, respectively, it increases with the reduction of the scale size, but the change range is small. It can be seen from Fig. 5 that there is a clear change on the

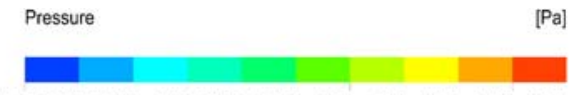

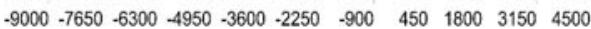
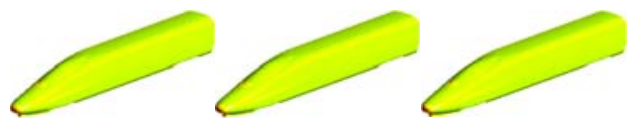

(a) $\lambda=1 / 1$

(b) $\lambda=1 / 2$

(c) $\lambda=1 / 8$
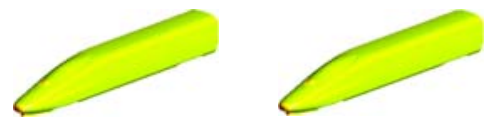
(e) $\lambda=1 / 16$
(f) $\lambda=1 / 25$

Fig. 5. Pressure distribution on the surface of the head car at different scale size. 
nose and streamlined part of the head car. To dictate the effect on the surface pressure in detail, the surface pressure at a cut plane $y=0 \mathrm{~m}$ of the head car is selected for analysis. Figure 6 shows the pressure distribution of the head car.

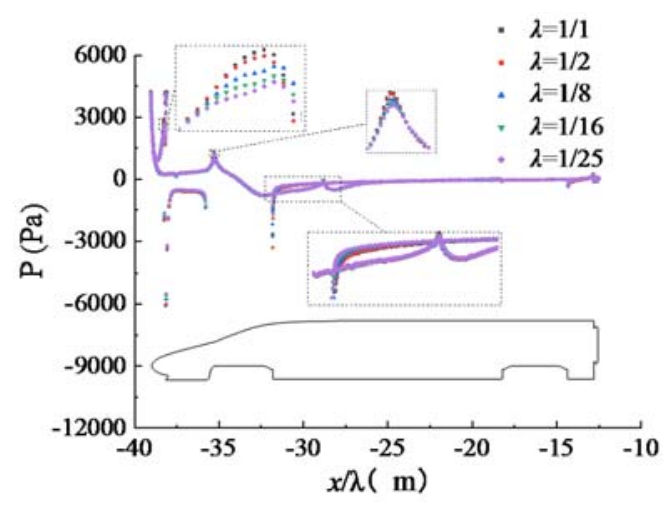

Fig. 6. Pressure distribution of the head car at a cut plane $y=0 \mathrm{~m}$.

The surface pressure shows different trends in different positions with the scale size changes. At the positions of $x / \lambda=-35.3 \mathrm{~m}$ and $x / \lambda=-28.8 \mathrm{~m}$ of the train surface, the pressure are $1201 \mathrm{~Pa}$ and $-95 \mathrm{~Pa}$ when the scale size $\lambda$ is $1 / 1$. Compared to the $1 / 25^{\text {th }}$ scaled train, the pressure of those positions increases by $113 \mathrm{~Pa}$ and $95 \mathrm{~Pa}$, respectively. The reduction of pressure at the head nose will weaken the pressure drag force of the train. At the position of $x=-29 \mathrm{~m}$. the surface pressure tends to increase as the scale size decreases. The variation of pressure in the rest of the head car is small and has less influence on the aerodynamic force.

Figure 7 shows the surface pressure of the tail car at different scale sizes. The surface pressure of the tail car is mostly negative. The pressure of the tail car's longitudinally symmetric mid-section is also extracted to explore the variation of the tail car's surface pressure. Figure 8 shows the pressure distribution at a cut plane $y=0 \mathrm{~m}$ of the tail car. Figure 8 shows the scale size has a larger effect on the tail car compared to the head car and middle car. In the longitudinally symmetric mid-section of the tail car at the position of $x / \lambda=35 \mathrm{~m}$ and $32 \mathrm{~m}$, the surface pressure are local maximum and local minimum, respectively. At the former positions, when the scale size $\lambda=1 / 25$, the absolute value of pressure change is $150 \mathrm{~Pa}$ compared to the full-size train. At the position of local maximum, the surface pressure decreases as the scale size decreases; at the position of local minimum, the surface pressure increases as the scale size decreases. It can be concluded that the smaller the scale size, the smaller the pressure gradient.

The relation between the surface pressure and the surrounding flow field is close. Figure 6 and Fig. 8 show the reasons for the change of train surface, and they can be attributed to the following two situations:
(1) Change in the position of the flow separation point (2) Change in velocity distribution of the boundary layer.

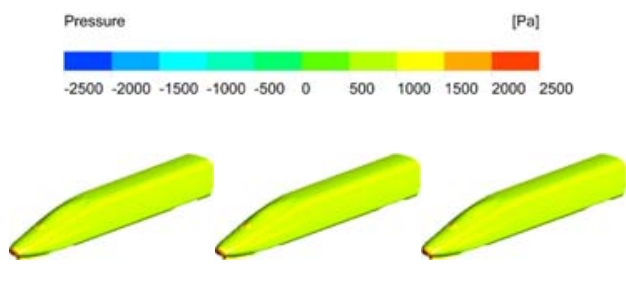
(a) $\lambda=1 / 1$
(b) $\lambda=1 / 2$
(c) $\lambda=1 / 8$

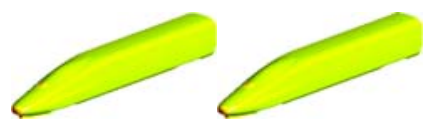
(e) $\lambda=1 / 16$
(f) $\lambda=1 / 25$

Fig. 7. Surface pressure on the surface of the tail car at different scale size.

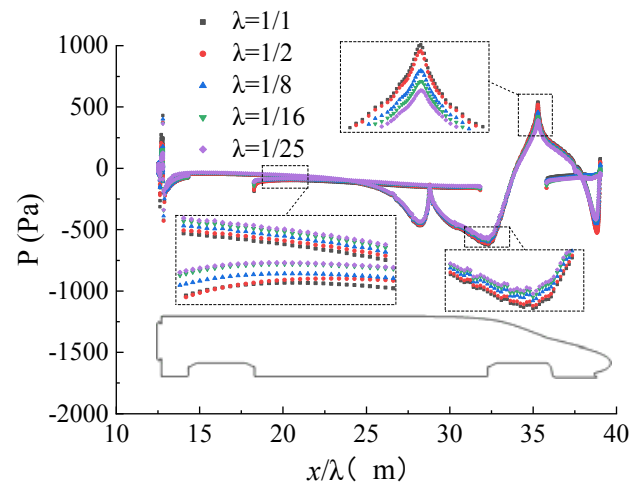

Fig. 8. Pressure distribution of the tail car at a cut plane $y=0 \mathrm{~m}$.

Figure 9 shows the flow field around the head nose at different scale sizes. The position of the flow separation point keeps moving toward the nose point of the head car which results in the reduction of pressure when the scale size decreases. Figure 10 shows the distribution of the flow field around the tail nose. The flow direction of the flow around the tail nose does not change, but the velocity gradient changes significantly. The pressure gradient around the train decreases with the reduction of the scale size The velocity range of the boundary layer is generally defined as $(0 \sim 0.99) \quad u_{\infty}$ and the boundary layer thickness $\sigma$ is defined as the distance from the point on the train surface along the vertical direction to the $0.99 u_{\infty}$ point. Figure $10(\mathrm{~g})$ shows the velocity distributions of $0.99 u_{\infty}$ at the cut plane of $x / \lambda=35 \mathrm{~m}$, it can be seen that the value of $\sigma / \lambda$ increases as the scale size decreases, indicating a decrease in the corresponding velocity gradient. 

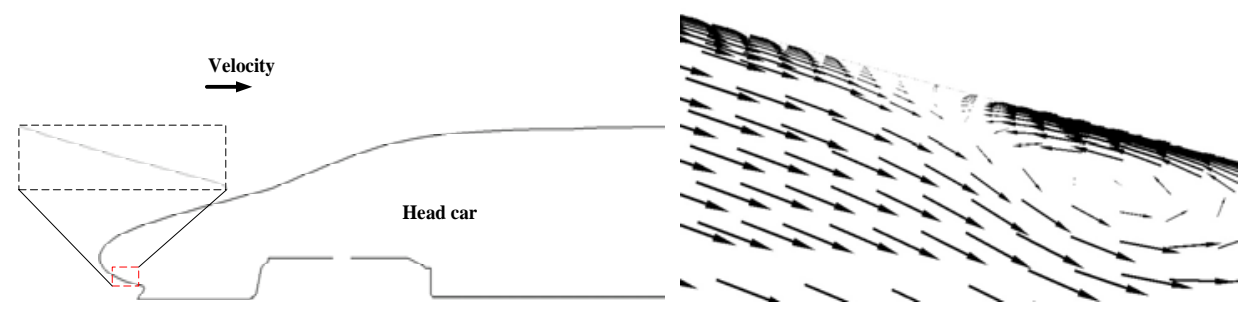

(a) Head car

(b) $\lambda=1 / 1$

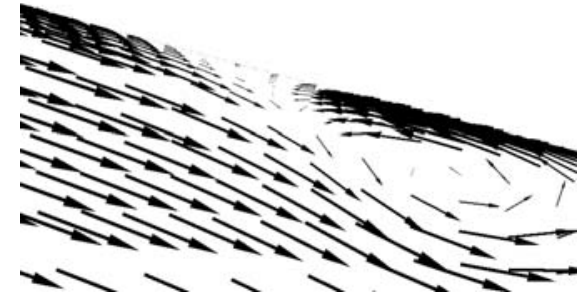

(c) $\lambda=1 / 2$

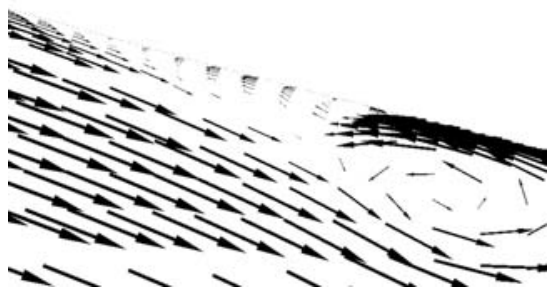

(e) $\lambda=1 / 16$

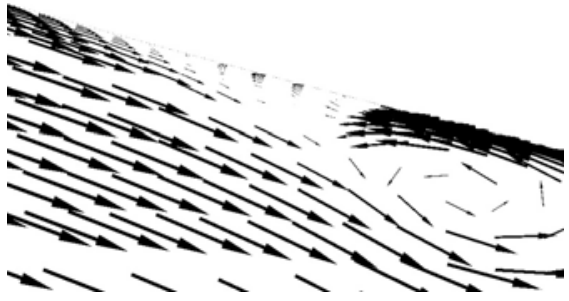

(d) $\lambda=1 / 8$

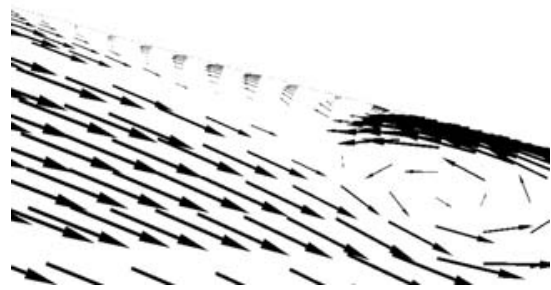

(f) $\lambda=1 / 25$

Fig. 9. Flow field around the nose point of the head car.

\subsection{Boundary layer}

At most zones of the train, the surface is like a flat plate. Therefore, reference can be made to the flow between two-dimensional flat plates, for which the Newtonian viscous force equation is

$\tau=\mu \frac{d u}{d y}$

where $\tau$ is the viscous shear stress, $\mu$ is the dynamic viscosity, and $d u / d y$ is the velocity gradient or shear strain rate. The magnitude of the viscous shear stress of the wall is determined by the velocity gradient of the viscous sublayer within the turbulent boundary layer.

The velocity distribution of the boundary layer is shown in Fig. 11. There is no significant change in the velocity distribution on the large scale except for the equipment bay. Two points are taken on the streamlined and non-streamlined parts of the longitudinally symmetric mid-section of the tail car. The position and value of $x / \lambda$ for the specific position are shown in Fig. 12(a). The boundary layer thicknesses at points a and $\mathrm{b}$ are defined as $\sigma_{a}$ and $\sigma_{b}$, respectively, and Fig. 12(b) shows the values of $\sigma_{a}$ and $\sigma_{b}$ for different scaled trains. There is a linear correlation between the thickness of boundary layer and the scale size, and the smaller the scale size, the thinner the thickness of the boundary layer. As the scale size increases, the boundary layer thickness increases.

When the scale size is changed, the viscosity of air and velocity of incoming flow are constant. According to Eq. (3), the value of the viscous force is inversely proportional to the thickness of the boundary layer. Figure 13 shows the viscous shear stress of $x$-direction at a cut plane $y=0 \mathrm{~m}$. When the scale size decreases, the viscous shear stress of $x$ direction tends to increase. The trend of the viscous force with the scale size is different from that of the boundary layer, which is due to the complex internal structure of the boundary layer. The internal structure of turbulent boundary layer is complex, including the linear layer, logarithmic law layer and transition layer; the changes within each sub-layer are not the same. 


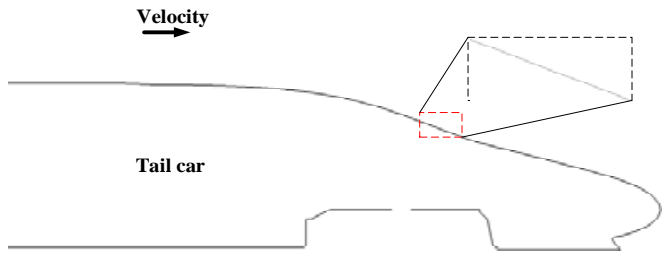

(a) Tail nose

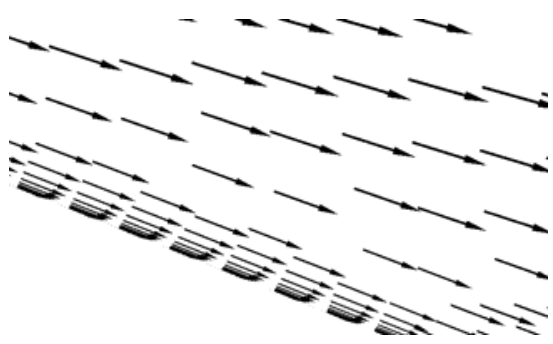

(c) $\lambda=1 / 2$

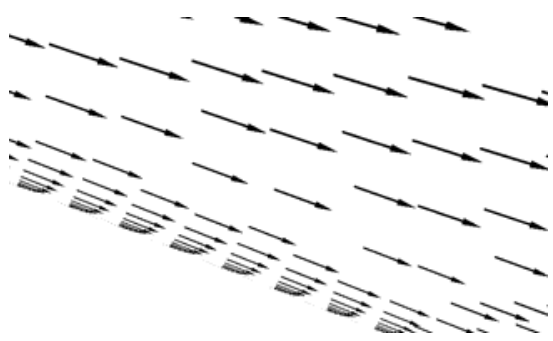

(e) $\lambda=1 / 16$

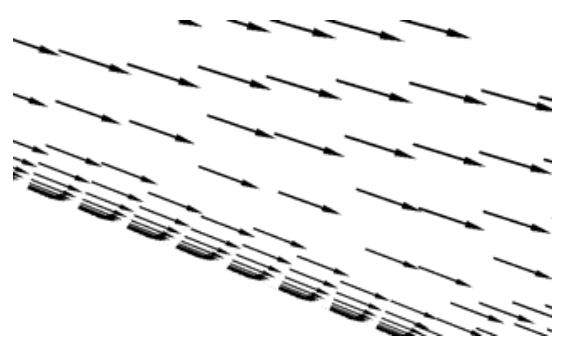

(b) $\lambda=1 / 1$

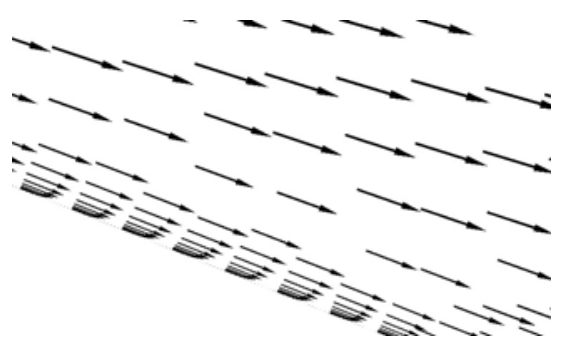

(d) $\lambda=1 / 8$

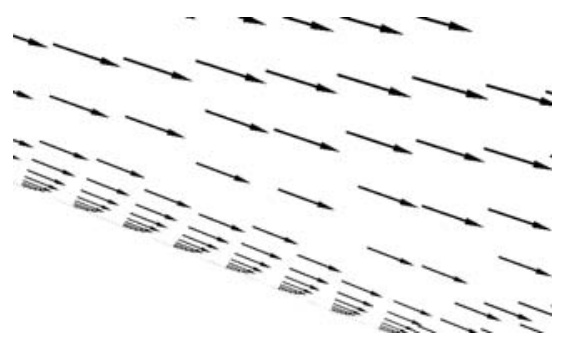

(f) $\lambda=1 / 25$

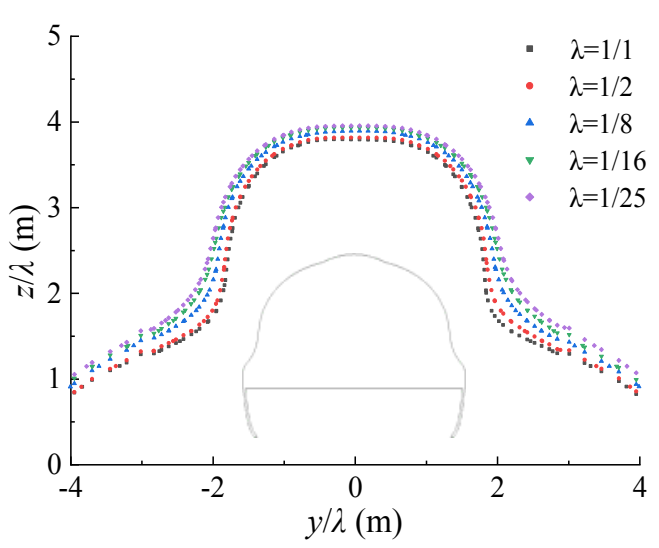

(g) Velocity distribution at different scale sizes

Fig. 10. Flow field around the tail nose.

\subsection{Aerodynamic characteristics}

The aerodynamic force consists of two parts pressure force and viscous force. The pressure force is the force caused by the pressure on the surface of the object, and the windward side, the shape and the position of the object in the airflow have a great influence. Viscous force is the force generated by the air viscosity. In the wind tunnel test, the aerodynamic drag coefficient and lift coefficient of the train are the main concern, and the effect of the scale size on the aerodynamic drag coefficient and lift coefficient will be discussed. 
C. Chang et al. / JAFM, Vol. 15, No. 1, pp. 209-220, 2022

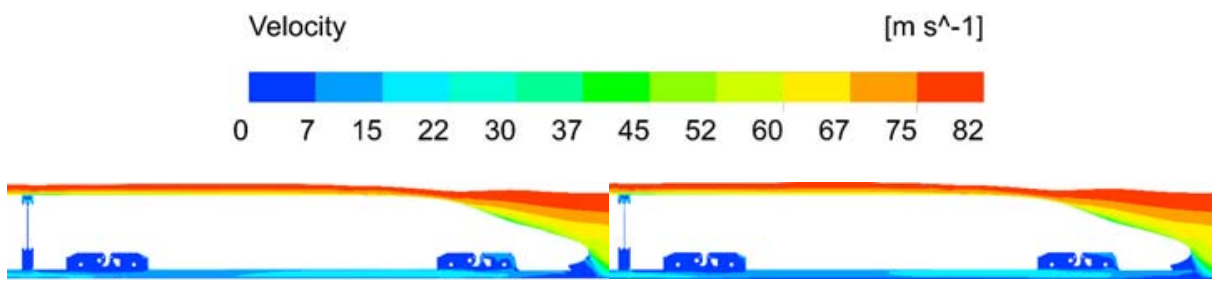
(a) $\lambda=1 / 1$
(b) $\lambda=1 / 2$

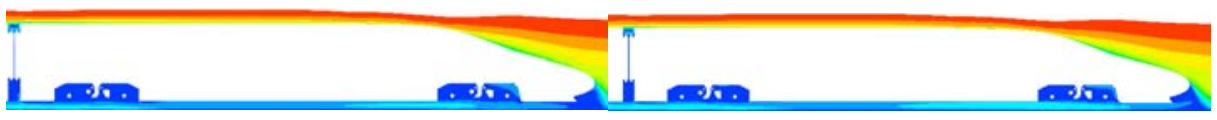

(d) $\lambda=1 / 8$

(b) $\lambda=1 / 16$

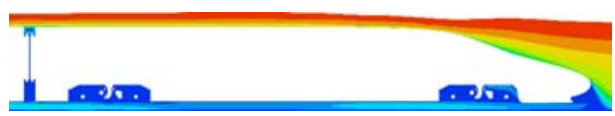

(e) $\lambda=1 / 25$

Fig. 11. Velocity distribution in the boundary layer at the mid-section of the tail car.

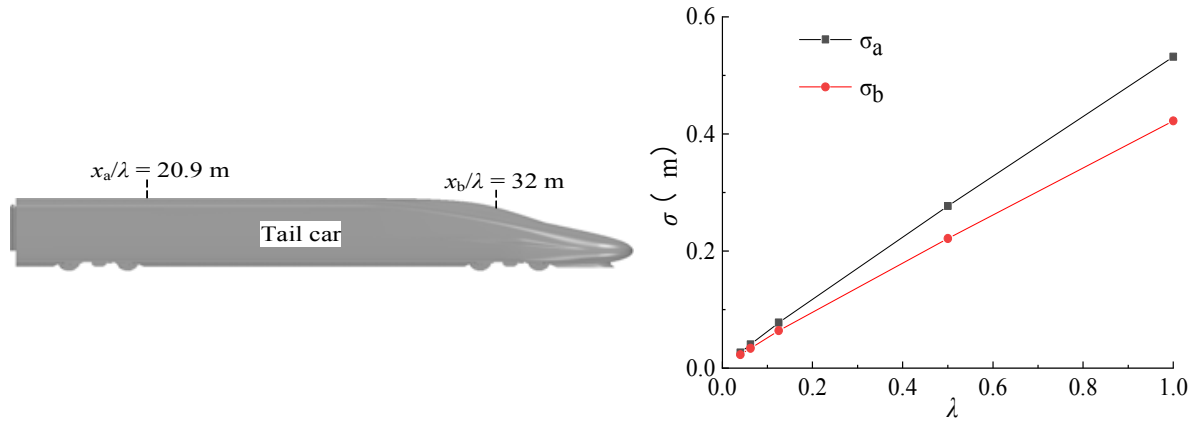

(a) Schematic diagram of selected points

(b) Boundary layer thickness

Fig. 12. Values of $\sigma_{a}$ and $\sigma_{b}$ at different scale sizes.

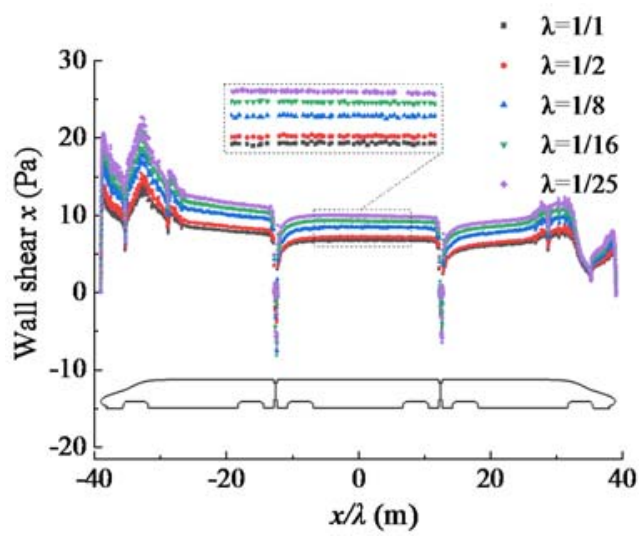

Fig. 13. Viscous shear stress at a cut plane $y=$ 0m.

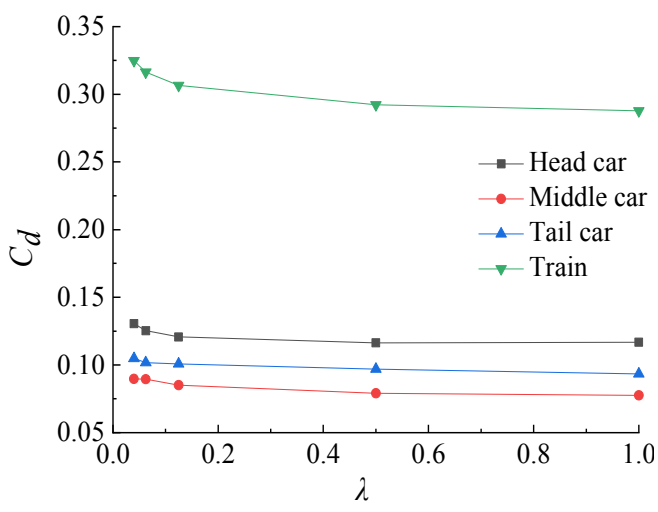

Fig. 14. Aerodynamic drag coefficients of the train at different scale sizes. 


\subsubsection{Aerodynamic drag coefficient}

Figure 14 shows the aerodynamic drag coefficients of the train at different scale sizes. The aerodynamic drag coefficients of the head car, middle car and tail car gradually increase as the scale size decreases. The drag coefficient of the train at the scale size $\lambda=1 / 25$ is 0.3249 , which is $12.92 \%$ larger than that of the full- size train, and the drag coefficients of the head, middle and tail car are $11.6 \%, 15.6 \%$ and $12.2 \%$ larger, respectively.

Figure 15 shows the results of pressure drag coefficients and viscous drag force coefficients for the head car, middle car and tail car at different scale sizes.

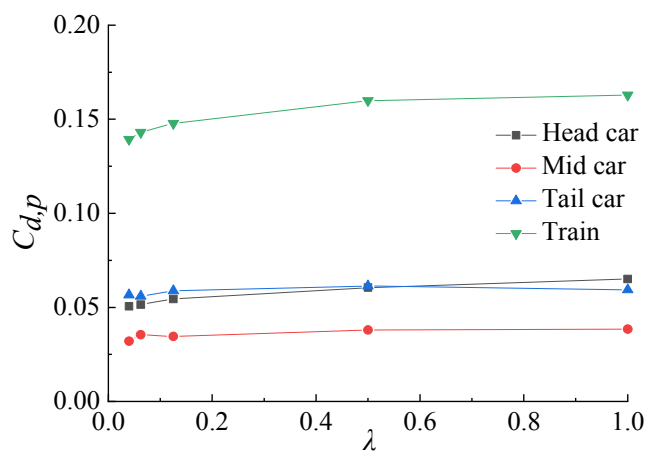

(a) Pressure drag coefficient

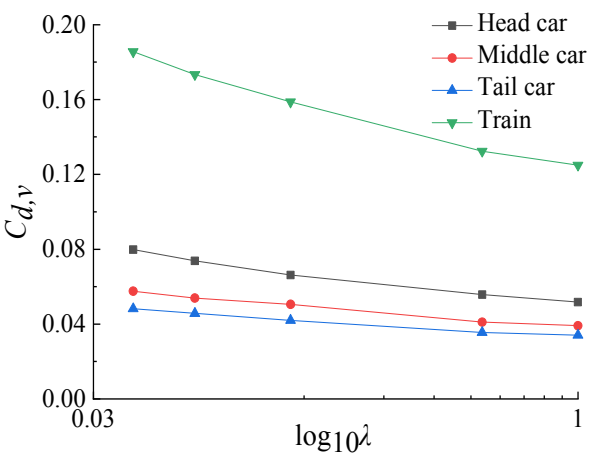

(b) Viscous drag coefficient

Fig. 15. Pressure drag coefficient and viscous drag coefficient of the train at different scale sizes.

The pressure drag force coefficient of the full-size train is 0.1831 , which accounts for $60 \%$ of the aerodynamic drag force coefficient of the train. When the scale size decreases, pressure drag force coefficients of the head car, middle car and tail car show a decreasing trend. When the scale size $\lambda=1 / 25$, the drag coefficient of the train is 0.164 , which is $11.4 \%$ smaller than that of the full-size train, and the head car, middle car and tail car are reduced by $15.3 \%$, 11.8 and $4.7 \%$ respectively.

The effect of viscous drag force is also not negligible. When the scale size $\lambda=1 / 25$ the viscous drag coefficient of the train is 0.186 , accounting for $57.1 \%$ of the aerodynamic drag coefficients. It can be seen from Fig. 15(b) that the viscous drag coefficients of the head car, middle car and tail car all increase gradually with the reduction of the scale size. It can be seen that the train viscous force of $x$-direction increases with the decrease of the scale size from the analysis of the boundary layer, therefore, it leads to the change of viscous drag coefficient as shown in Fig. 15(b). The viscous drag coefficients are generally related to $\log _{10} \mathrm{Re}$ in a power exponential relationship(Baker and Brockie 1991). The viscous drag coefficients of the head car, middle car, tail car and train are fitted and the fitted expressions are

$$
\begin{aligned}
& \mathrm{C}_{d, v \text {-head }}=7.72348\left(\log _{10} \mathrm{Re}\right)^{-2.36745} \\
& \mathrm{C}_{\mathrm{d}, v \text {-middle }}=3.78482\left(\log _{10} \mathrm{Re}\right)^{-2.16214} \\
& \mathrm{C}_{d, v \text {-tail }}=2.15844\left(\log _{10} \mathrm{Re}\right)^{-1.96481} \\
& \mathrm{C}_{d, v \text {-train }}=12.95557\left(\log _{10} \mathrm{Re}\right)^{-2.19585}
\end{aligned}
$$

The $\mathrm{R}^{2}$ of the fit was $0.9956,0.9906,0.9950$ and 0.9974 , respectively, and the fit results were good.

\subsubsection{Aerodynamic lift force coefficient}

Figure 16 shows the aerodynamic lift coefficients and pressure lift coefficients for the head car, middle car and tail car at different scale sizes. The aerodynamic lift coefficients of the train show an increasing trend as the scale size decreases. When the scale size is reduced from $\lambda=1 / 1$ to $\lambda=1 / 25$, the aerodynamic lift coefficients of the head car and the tail car change $15.42 \%$ and $5.72 \%$, respectively. When the scale size $\lambda=1 / 25$, the pressure coefficients of the head car, middle car, and tail car are -0.054 , 0.0065 , and 0.096 , respectively. Compared to the full-size train, the pressure coefficients of the head car, middle car, and tail car change $16.1 \%, 46.6 \%$ and $12.3 \%$. The viscous lift force is concentrated on the vertical surface of the windshield section of the train, where the speed is small leading to a small viscous lift force. Figure 17 shows the results of viscous lift coefficients for the train with different scale sizes. The absolute values of the viscous lift coefficients of the head car and tail car increase gradually with the decrease of the scale size. Since the viscous lift coefficient of the middle car is determined by the velocity distribution at the windshield, when the scale size $\lambda=1 / 25$, the direction of the viscous lift force of the middle car even changes.

\subsubsection{Aerodynamic characteristic in crosswinds}

In the case of crosswind, the aerodynamic force change significantly, There it is very import to study the aerodynamic characteristics of high-speed trains in crosswind (Li 2019b). Table 3 shows the pressure 


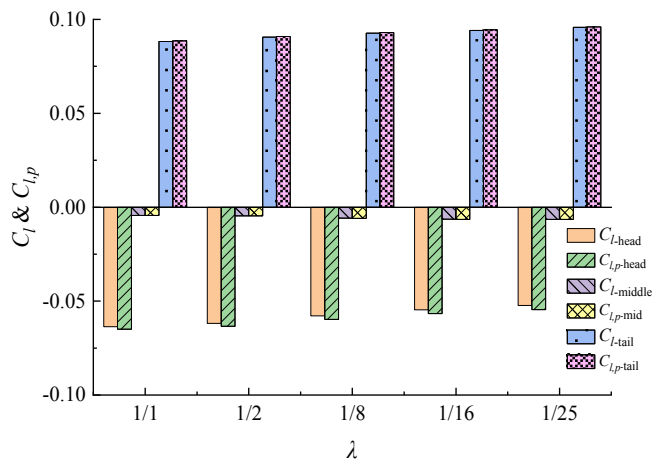

Fig. 16. Train aerodynamic lift coefficient and pressure lift coefficient at different scale sizes.

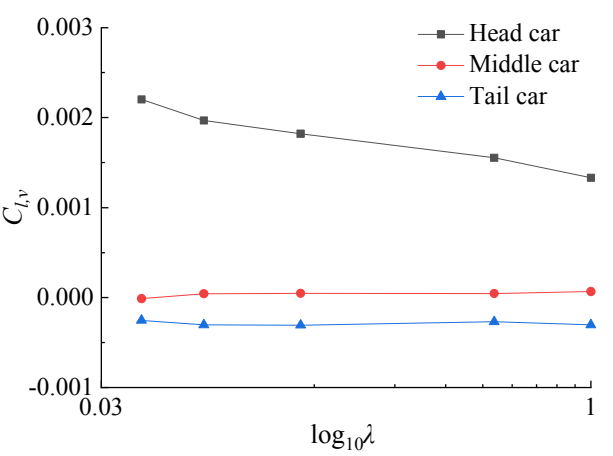

Fig. 17. Viscous lift coefficients of the train at different scale sizes.

Table 3 Aeodynamic drag coefficients of the train at different scale sizes

\begin{tabular}{|c|c|c|c|c|c|c|c|c|c|}
\hline$\Lambda$ & $\mathrm{C}_{d, p \text {-head }}$ & $\begin{array}{c}\mathrm{C}_{d, p-} \\
\text { middle }\end{array}$ & $\mathrm{C}_{d, p \text {-tail }}$ & $\mathrm{C}_{d, v \text {-head }}$ & $\begin{array}{c}\mathrm{C}_{d, v-} \\
\text { middle }\end{array}$ & $\mathrm{C}_{d, v \text {-tail }}$ & $\mathrm{C}_{d \text {-head }}$ & $\mathrm{C}_{d \text {-middle }}$ & $\mathrm{C}_{d \text {-tail }}$ \\
\hline $1 / 1$ & 0.0172 & 0.1350 & 0.1540 & 0.0639 & 0.0542 & 0.0475 & 0.0811 & 0.1892 & 0.2015 \\
\hline $1 / 2$ & 0.0184 & 0.1250 & 0.1580 & 0.0689 & 0.0579 & 0.0493 & 0.0873 & 0.1829 & 0.2073 \\
\hline $1 / 8$ & 0.0227 & 0.1240 & 0.1320 & 0.0791 & 0.0683 & 0.0586 & 0.1018 & 0.1923 & 0.1906 \\
\hline $1 / 16$ & 0.0235 & 0.1200 & 0.1240 & 0.0879 & 0.0744 & 0.0627 & 0.1114 & 0.1944 & 0.1867 \\
\hline $1 / 25$ & 0.0261 & 0.1130 & 0.1210 & 0.0942 & 0.0825 & 0.0663 & 0.1203 & 0.1955 & 0.1873 \\
\hline
\end{tabular}

drag coefficients, viscous drag coefficients and aerodynamic drag coefficients at different scale sizes. Compared to the open-air operating condition, the trend of the pressure drag coefficients and viscous drag coefficients are consistent except for the head car. Crosswinds will cause flow separation on the leeward side of the head car, and the surface pressure of the head car changes differently from the open-air operating condition. Meanwhile, the length of the train along the wind direction is reduced in crosswinds, which makes the increments of viscous drag coefficients less with the scale size decrease. The change of the aerodynamic drag coefficients is jointly determined by the pressure drag and viscous drag coefficients. Compared to the full-size train, when the scale size $\lambda=1 / 25$, the aerodynamic drag coefficients of the head car and middle car increase $48.2 \%$ and $3.44 \%$, respectively. However, the aerodynamic drag coefficient of the tail car decreases by $0.47 \%$.

\section{Conclusion}

(1) The position of the flow separation point and the velocity distribution near the train wall will be affected by the scale size: when the scale size decreases, the position of the flow separation at the streamlined part of the head car moves toward the head car's nose point, leading to a reduction in pressure; the velocity gradient near the train wall gradually decreases as the scale size decreases, which means that the pressure gradient at the corresponding position also gradually decreases.
(2) As the velocity of the incoming flow and the viscosity of air are constant, the thickness of the boundary layer can be used to measure the wall shear stress according to Eq. (3). With the reduction of the scale size, the thickness of the boundary layer decreases, and there is an approximately linear relationship between the thickness of the boundary layer and the scale size.

(3) The aerodynamic force is the result of the combined effect of pressure and viscous force. The influence of pressure drag and viscous drag cannot be ignored. As the scale size decreases, the pressure drag coefficient tends to decrease and the viscous drag coefficient tends to increase. Compared with the full-size train, when the scale size $\lambda=1 / 25$, the pressure drag coefficient of the train decreases by 0.0235 , a change of $14.4 \%$, and the viscous drag coefficient increases by 0.0607 , a change of $48.56 \%$. The aerodynamic lift is mainly the pressure lift, the influence of viscous lift is small and ignorable. The train aerodynamic lift coefficient increases with the reduction of scale size, and the head car and tail car aerodynamic lift coefficients decrease by $15.42 \%$ and $5.72 \%$ respectively.

(4) Compared to the open-air operating condition, the trend of the pressure drag coefficients and viscous drag coefficients is consistent except for the head car in crosswind operating condition when the scale size decreases. The length of the train along the wind direction is reduced in crosswinds, and the increments of viscous drag coefficients are less. Compared to the full-size train, when the scale size 
$\lambda=1 / 25$, the aerodynamic drag coefficients of the head car and middle car increase $48.3 \%$ and $3.3 \%$, respectively. However, the aerodynamic drag coefficient of the tail car decreases by $0.7 \%$.

\section{ACKNOWLEDGEMENTS}

This work was supported by National Key Research and Development Program of China (2020YFA0710902), National Natural Science Foundation of China (12172308), Fundamental Research Funds for the Central Universities (2682021ZTPY124) and State Key Laboratory of Traction Power (2019TPL_T02).

\section{REFERENCES}

Baker, C. J. and N. J. Brockie (1991). Wind tunnel tests to obtain train aerodynamic drag coefficients: Reynolds number and ground simulation effects. Journal of Wind Engineering and Industrial Aerodynamics 38(1), 23-28.

Bell, J. R., D. Burton, M. C. Thompson, A. H. Herbst and J. Sheridan (2017). A wind-tunnel methodology for assessing the slipstream of high-speed trains. Journal of Wind Engineering and Industrial Aerodynamics 166, $1-19$.

Bell, J. R., D. Burton, M. Thompson, A. Herbst and J. Sheridan (2014). Wind tunnel analysis of the slipstream and wake of a high-speed train. Journal of Wind Engineering and Industrial Aerodynamics 134(1), 122-138.

Catalano, P., M. Wang, G. Iaccarino and P. Moin (2003). Numerical simulation of the flow around a circular cylinder at high Reynolds numbers. International Journal of Heat and Fluid Flow 24(4), 463-469.

Collin, C., S. Mack, T. Indinger and J. Mueller (2016). A numerical and experimental evaluation of open jet wind tunnel interferences using the DrivAer reference model. SAE International Journal of Passenger Cars-Mechanical Systems 9(2), 657-679.

De Graaff, D. B. and J. K. Eaton (2000). Reynoldsnumber scaling of the flat-plate turbulent boundary layer. Journal of Fluid Mechanics 422, 319-346.

Emil, L., S. Sebben and A. Broniewicz (2020). Inclusion of the physical wind tunnel in vehicle CFD simulations for improved prediction quality. Journal of Wind Engineering and Industrial Aerodynamics 197, 104055.

Han, Y. D. and Y. Song (2017). Scale effect analysis in aerodynamic performance of highspeed train. Journal of Zhejiang University (Engineering Science) 51(12), 2383-2391.
Kwon, H. B., Y. W. Park, D. H. Lee and M. S. Kim (2001). Wind tunnel experiments on Korean high-speed trains using various ground simulation techniques. Journal of Wind Engineering and Industrial Aerodynamics 89(13), 1179-1195.

Li, T., H. Hemida, M. M. Rashidi and W. Zhang (2020). The effect of numerical divergence schemes on the flow around trains. Fluid Dynamics Research 52(2), 025509.

Li, T., J. Y. Zhang, M. Rashidi and M. Yu (2019a). On the Reynolds-averaged Navier-Stokes modeling of the flow around a simplified train in crosswinds. Journal of Applied Fluid Mechanics 12(2), 551-563.

Li, T., Q. Deng, J.Y. Zhang (2019b). Effect of RANS turbulence model on aerodynamic behavior of trains in crosswind. Chinese Journal of Mechanical Engineering 32(1), 85

Li, T., Z. Dai, M. Yu and W. Zhang (2021). Numerical investigation on the aerodynamic resistances of double-unit trains with different gap lengths. Engineering Applications of Computational Fluid Mechanics 15(1), 549-560.

Li, W. H., T. H. Liu, J. Zhang, Z. W. Chen, X. D. Chen and T. Z. Xie (2017). Aerodynamic study of two opposing moving trains in a tunnel based on different nose contours. Journal of Applied Fluid Mechanics 10(5), 1375-1386.

Lu, C. L., Q. S. Li, S. H. Huang, F. B. Chen and X. Y. $\mathrm{Fu}$ (2012). Large eddy simulation of wind effects on a long-span complex roof structure. Journal of Wind Engineering and Industrial Aerodynamics 100(1), 1-18.

Munoz, P. J., J. Garcia and A. Crespo (2015) Aerodynamic optimization of the nose shape of a train using the adjoint method. Journal of Applied Fluid Mechanics 8(3), 601- 612.

Niu, J., X. Liang and D. Zhou (2016). Experimental study on the effect of Reynolds number on aerodynamic performance of high-speed train with and without yaw angle. Journal of Wind Engineering and Industrial Aerodynamics 157, 36-46.

Paz, C., E. Suárez, C. Gil and M. Concheiro (2015). Numerical study of the impact of windblown sand particles on a high-speed train. Journal of Wind Engineering and Industrial Aerodynamics 145, 87-93.

Vallikivi, M., M. Hultmark and A. J. Smits (2015). Turbulent boundary layer statistics at very high Reynolds number. Journal of Fluid Mechanics 779, $371-389$.

Xia, Y., T. Liu, H. Gu, Z. Guo, Z. Chen, W. Li and L. Li (2020). Aerodynamic effects of the gap spacing between adjacent vehicles on wind tunnel train models. Engineering Applications 
C. Chang et al. / JAFM, Vol. 15, No. 1, pp. 209-220, 2022

of Computational Fluid Mechanics 14(1), 835852.

Yu, M., J. Liu and Z. Dai (2021). Aerodynamic characteristics of a high-speed train exposed to heavy rain environment based on non-spherical raindrop. Journal of Wind Engineering and Industrial Aerodynamics 211, 104532.

Zhang, J., K. He, X. Xiong, J. Wang and G. Gao (2017). Numerical simulation with a DES approach for a high-speed train subjected to the crosswind. Journal of Applied Fluid Mechanics 10(5), 1329-1342.

Zhou, D., H. Q. Tian, J. Zhang and M. Z. Yang (2014). Pressure transients induced by a high-speed train passing through a station. Journal of Wind Engineering and Industrial Aerodynamics 135, 1-9. 